E. Krebs

Im Grunde genommen sollten sich alle Einwohner unseres Landes für den Schutz des Waldes einsetzen, denn sie alle zehren direkt oder indirekt, bewußt oder unbewußt von den mannigfachen Leistungen, die der Wald vollbringt. Abgesehen von der wirtschaftlichen Bedeutung erfüllt er in steigendem Umfang Schutz- und Wohlfahrtsaufgaben.

Die Wälder schützten in den Bergtälern seit jeher die Siedlungen, landwirtschaftlichen Güter und Verkehrswege vor den vielen Gefahren der Gebirgsnatur. Unsere Bergtäler wären ohne Wald gar nicht bewohnbar. Der über den Dörfern liegende Wald wurde daher schon vor Jahrhunderten in Bann gelegt; er war heilig, und es war eines der größten Verbrechen, im Bannwald Bäume zu schänden. Daher ist das Verständnis für den Wald und die Liebe zum Wald im Bewußtsein der Bergbevölkerung besonders tief verankert, und Schutzwälder im traditionellen Sinn waren ursprünglich die Wälder der Alpen, Voralpen und des Jura.

Die Entwicklung der jüngsten Zeit gab nun aber auch den Wäldern außerhalb der Bergtäler oder gerade den Wäldern in der dicht besiedelten und stark industrialisierten Zone des Mittellandes erhöhte Bedeutung. Die Bevölkerung der Schweiz ist innert 100 Jahren ungefähr zweieinhalb mal größer geworden. Dabei ist eine starke Zusammenballung in großen Industrieortschaften und in Städten festzustellen, und während die Bevölkerungsdichte in der Schweiz bis heute auf rund 150 Einwohner auf 1 Quadratkilometer angewachsen ist,beträgt sie für das Mittelland rund 250, für die Stadt Zürich 5000, für Basel 9000 und für Genf sogar 11 000. In Zusammenhang damit haben sich die geschlossenen Siedlungs- und Industriezonen ausgedehnt. Der Bürger der alten Stadt fand früher vor den Toren weite Landwirtschaftsgebiete, Grün- und Erholungsflächen; diese sind heute vom Häusermeer überflutet. Die in weiterem Umkreis liegenden kleinen, friedlichen Bauerndörfer sind von der Stadt aufgefressen worden, sind im Häusermeer untergegangen. Der Motorfahrzeugbestand der Schweiz ist in der Zeit seit 1910 von rund 7000 auf 1200000 angewachsen. In einem halben Jahrhundert ist er $170 \mathrm{mal}$, im Kanton Zürich 200mal größer geworden. In der gleichen Zeit hat sich der Wasserverbrauch vervierfacht.

Durch diese Entwicklung haben sich die Lebensumstände unserer Bevölkerung in mancher Hinsicht progressiv verschlechtert. Motorisierung, Industrialisierung, Lärm und Betrieb bringen eine unerhörte physische und psychische Belastung der Menschen. Die Arbeitswege wurden länger, die Ruhezeiten werden immer weniger für eine sinnvolle Erholung verwendet. Die Luft wird in steigendem Maß verstaubt und vergiftet, und die Verschmutzung der Oberflächengewässer geht trotz aller Maßnahmen in beängstigendem Umfang weiter.

Diese Entwicklung brachte uns neuartige Gefahren, die wir uns selbst eingebrockt haben durch die brutalen Eingriffe in den Haushalt der Natur und durch die Zerstörung der natürlichen Lebensgrundlagen. Damit erhielt der Wald besonders in den dicht bewohnten Gebieten des Mittellandes eine ungeahnte Bedeutung und eine erhöhte Wertschätzung. Da er in der Lage ist, viele dieser Gefahren zu mildern oder sogar zu beseitigen, erfüllt er schon heute, noch mehr aber in Zukunft für die Erholung, Gesundheit und das Wohlergehen lebensentscheidende Aufgaben.

\section{Wald und Wasser}

Der Waldboden, der nie bearbeitet wird, hat eine großartige Architektur. Seine lockere Struktur wird durch zahllose Hohlräume, Wurzelkanäle, Mausund Wurmgänge und die kleinen Lufträume zwischen den Krümeln der Walderde gebildet. Der Anteil an Hohlräumen und Poren macht bei gutem, gewachsenem Waldboden bis zur Hälfte oder sogar bis drei Viertel des Bodenvolumens aus. Der Waldboden ist daher 20 bis 30 mal durchlässiger als Freilandboden. Das hat zur Folge, daß im Wald selbst an steilen Hängen alles Meteorwasser praktisch restlos in den Boden eindringt. Im offenen Land fließt wegen der geringeren Durchlässigkeit bei starken Niederschlägen und rascher Schneeschmelze ein großer Teil oberflächlich ab. Dieser starke Oberflächenabfluß bewirkt Abschwemmung der guten Erde, Unterspülung der Hänge und Rutschungen im Oberlauf und Hochwasser, Überschwemmungen und Materialablagerung im Unterlauf. Nach den langjährigen Untersuchungen von Engler und Burger ist die Hochwassermenge eines Flusses bei guter Bewaldung des Einzugsgebietes kaum halb so groß wie jene von Gewässern bei schwacher Bewaldung, während aber umgekehrt der Abfluß während 
der nachfolgenden Trockenzeiten mehrfach größer ist. Der Wald gleicht den Wasserhaushalt der Gewässer stark aus, bricht die gefährlichen Hochwasserspitzen, verzögert den Wasserabflu $\beta$ zeitlich, schützt den Boden vor Auswaschung und verbessert den Wasserstand in Trockenzeiten bedeutend.

Dieser günstige Einfluß des Waldes auf den Stand der Gewässer wurde von Forstleuten und Naturwissenschaftern schon erkannt, lange bevor die Forschung sich mit diesen Fragen befaßte. Die Natur selbst ist ein großartiges Experimentierfeld und demonstrierte die Folgen der früheren Waldzerstörung auch in unserem Lande mit eindrücklicher Gewalt. Die zunehmende Entwicklung der Bergtäler und die Mißwirtschaft durch Kahlschläge führten unweigerlich zu Hochwasser, Bodenzerstörung und Rutschungen im Oberlauf und zu Überschwemmungen und Geschiebeablagerungen im Mittel- und Unterlauf.

Von besonderer Bedeutung ist sodann der Einfluß des Waldes auf die Wassergüte. Der mit einer guten Laub- und Nadelstreu und einer starken Humusschicht überdeckte Waldboden ist ein ausgezeichneter Filter für Meteorwasser. Quell- und Grundwasser aus gut bewaldeten Gebieten ist daher noch absolut gesund, während unsere Oberflächengewässer in bedenklichem $\mathrm{Ma} ß$ verschmutzt und verseucht sind. Durch ausgedehnte Bewaldung wird auch jede Überbauung und damit jegliche Verunreinigung durch Zuleitung ungereinigter Abwässer, Versickerung von Öl oder Auswaschung landwirtschaftlicher Düngstoffe verhindert. In den Quellund Grundwassergebieten ist daher der Wald nicht nur vollumfänglich zu erhalten, sondern es drängt sich seine Ausdehnung durch Aufforstung auf.

\section{Wald und Luft}

Die Luftverschmutzung ist in vielen Gebieten viel schlimmer als man glaubt. Sie ist aber weniger augenfällig als die Gewässerverschmutzung und wird daher nur zu leicht übersehen.

In den Industrieballungsräumen hat die Luftverstaubung ein Ausmaß erreicht, daß auch bei schönem Wetter eine ständige Trübung besteht. Die amerikanischen Industriezentren schleudern jährlich 6 Millionen t Kohlenstaub in die Luft. Durch den heutigen Verkehr werden unvorstellbare Mengen Straßenschmutz, Gummi- und Asphaltstaub aufgewirbelt. Im ausgedehnten Industrieraum der Ruhr wird der Sonnenlichteinfall wegen der Dunstglocke um ungefähr $40 \%$ verkleinert. Blauer Himmel und eine strahlende Sonne sind etwas Unbekanntes; auch bei schönstem Wetter ist der Himmel grau und die Sonne verschleiert. In Düsseldorf lagern sich jährlich $12 \mathrm{~kg}$ Staub, im Zentrum des Ruhrgebietes $36 \mathrm{~kg}$ je Are Bodenfläche ab; in englischen Indu- striestädten hat man Staubablagerungen bis $60 \mathrm{~kg} /$ Are im Jahr gemessen.

Für die Klärung verstaubter Luft leisten Wälder und stark gegliederte Baumgürtel einen großartigen Beitrag. Das Kronendach des Waldes wirkt als Filter, durch den die Staubteilchen der durchströmenden Luft abgefangen werden. Nach Messungen von Meldau vermögen Föhren- und Rottannenbestände jährlich bis 35, Buchenbestände sogar bis 70 t Staub je Hektare aus der Luft auszufiltrieren. Im Waldinnern beträgt daher der Staubgehalt der Luft nur einen kleinen Bruchteil desjenigen der Luft in der Stadt oder über Industriegebieten. Wer atmet daher nicht tief auf, wenn er in die weiten Hallen eines Waldes tritt, um die Lunge mit der reinen, frischen Luft zu füllen?

Schwerwiegender ist das Problem der Luftvergiftung durch die Abgase der Verbrennungsöfen, Verbrennungsmotoren und der chemischen Industrie mit den vielseitigen Verbindungen von Schwefel, Fluor, Chlor und durch Kohlenmonoxyd und -dioxyd. Der Wald steht dieser Gefahr praktisch hilflos gegenüber, da er unter der Vergiftung mehr leidet, als er zur Verbesserung beizutragen vermag. Wohl besitzt der Wald ein gewisses Speichervermögen. In der Nähe von Industriewerken enthalten die Blätter und Nadeln einen zwei- bis dreifachen Gehalt an Giftstoffen gegenüber dem gesunden Wald. Die damit bewirkte Entgiftung der Luft vollbringt der Wald aber unter Aufopferung seiner Gesundheit. Besonders empfindlich sind die Nadelhölzer, die schon bei Gaskonzentrationen zugrunde gehen, die bei den Laubhölzern erst Zuwachsrückgänge verursachen. Man schätzt, daß in Nordrhein-Westfalen 80000 ha Wald unter Raucheinfluß leiden oder abgestorben sind. In den über 1000 ha großen Stadtwaldungen von Duisburg betrug der Anteil der standortsgemäßen Föhren früher rund ein Drittel; heute stehen auf dieser Fläche kaum noch 100 bis 200 Föhren. In der Nähe solcher Immissionszentren muß daher der Waldeigentümer eine starke Behinderung der Waldbewirtschaftung und schwerwiegende Ertragseinbußen in Kauf nehmen. Viel schlimmer ist, daß der Wald, der gerade in diesen Ballungsgebieten in allererster Linie Aufgaben des Gewässerschutzes, der Luftregenerierung, der Erholung und Gesundheit der Bevölkerung zu erfüllen hat, in seinem Bestand überhaupt in Frage gestellt ist.

Von besonderer Bedeutung ist der Wald sodann für den Sauerstoffhaushalt der Luft. Nur die grüne Vegetationsdecke und vor allem die Wälder sind in der Lage, die giftige Kohlensäure, die durch die Atmung der Lebewesen und durch die technische Verbrennung in riesigen Mengen erzeugt wird, wieder abzubauen und dabei gleichzeitig die Sauerstoffmengen wieder zu erzeugen, die durch die gleichen Prozesse verbraucht werden. Der Wald ist daher für 
die Regenerierung der Atemluft von unschätzbarem Wert.

\section{Wald und Landschaft}

Der Wald ist neben der Topographie und den Gewässern das beständigste Landschaftselement. $\mathrm{Er}$ prägt das Gesicht unserer Heimat. Durch großflächige Waldrodungen würde das vertraute Bild der Landschaft schärfer und schroffer umgestaltet, als das im Zeitalter der Baumaschinen durch Großbaustellen und die Ausdehnung der Siedlungen ohnehin erfolgt. Der Wald stellt auch zusammen mit dem Gelände ein festes Gerippe für die vernünftige Raumplanung und Raumnutzung dar. In der unaufhaltsamen Entwicklung ist der Wald der ruhende Pol. Auch wenn das stürmisch wachsende Häusermeer die Umgebung der Stadt überflutet, zwischen den Wäldern durchquillt und weit in die offene Landschaft hinausgreift, vor dem Wald muß es gezwungenermaßen Halt machen. Der Wald bildet damit die wirksamsten, billigsten und bleibenden Trenn- und Grüngürtel im Siedlungs- und Industrieraum, die für eine sinnvolle Gliederung unerläßlich sind. Müßten derartige Grün- und Erholungsflächen besonders geschaffen werden, dann wären angesichts der heutigen Bodenpreise auch die reichsten Gemeinwesen nicht in der Lage, auch nur kleine Teilflächen des bestehenden Waldes zu verwirklichen. Es ist daher vom Standpunkt der Orts- und Regionalplanung aus dringend nötig, nicht nur an der Waldfläche, sondern möglichst auch an der heute bestehenden Waldverteilung festzuhalten.

\section{Wald und Mensch}

Im Jahre 1900 lebten 22\% der Bevölkerung unseres Landes in Ortschaften mit über 10000 Einwohnern. 1962 waren es zwei Fünftel und um die nächste Jahrhundertwende herum werden es drei Fünftel sein. Das Erholungsbedürfnis wird angesichts der heutigen Lebensgewohnheiten immer größer. Wandern und Aufenthalt in frischer Luft werden damit stets wichtiger. Von besonderer Bedeutung sind die ortsnahen Wälder, die Ausspannung und Erholung auch am Feierabend und über das Wochenende ermöglichen.

Die Erholungswirkung des Waldes ist aus verschiedenen Gründen besonders groß. Uns packt die großartige Lebensgemeinschaft, die Gemeinschaft zahlloser großer und kleiner Bäume, der reichen Begleitflora, wo wohltuendes Grün herrscht und der ganze weite Raum von den tiefsten Wurzeln der Erde bis zu den äußersten Zweigen der Bäume mit Leben mannigfachster Art erfüllt ist; eine Gemeinschaft, in der alle Konstituenten durch tausendfa- che gegenseitige Beziehungen und Abhängigkelten miteinander verbunden sind, wo eine Stetigkeit und Harmonie herrscht, die uns innerlich ergreift. Uns erfreut das ziellose Wandern auf verschlungenen Wegen oder quer durch den Wald, das Recht, den Weg verlassen zu dürfen, um zwischen den Bäumen durchzugehen und die tausendfachen großen und kleinen Schönheiten und Wunder, das Spiel von Licht und Schatten zu erleben; es ist der Gegensatz zum täglichen, hastigen Gang auf den schnurgeraden Asphaltstraßen der lärmigen Stadt. Wir suchen im Wald auch die Ruhe, die Stille um uns und die Stille in uns. Es ist die Flucht vor dem Lärm. In der Ruhe und Einsamkeit des Waldes finden wir wieder den Weg zu uns selbst zurück, wir können uns mit unserem eigenen Ich befassen, wir vernehmen wieder einmal die Stimme unseres Herzens, all das, was sonst vom täglichen Betrieb und Geschehen übertönt wird. Auf einem solchen Gang durch Wald und Natur beginnen in uns Saiten zu schwingen, die vielleicht lange still waren. Wir spüren, daß im Leben noch etwas da ist, das über uns steht, das wir nicht beherrschen, das wir trotz aller Technik nicht zu lenken vermögen. Uns packt das Geheimnisvolle, das stille Walten der Natur, die ewige Erneuerung des Lebens, und uns erfüllt das tiefe, ethische Gefühl der Ehrfurcht vor dem Göttlichen. Die Spannungen lösen sich, wir atmen tief auf, wir erkennen, daß unsere Arbeit, Erfolg oder Mißerfolg, Verdienst und Vergnügen nicht das Wichtigste im Leben sind; manche Überheblichkeit fällt, mancher Ärger verfliegt, und manche Sorge schwindet. Wir kehren entspannt zurück, um mit frohem Mut die Aufgabe wieder aufzunehmen, die uns das Leben zuwies oder die wir uns selbst stellten.

\section{Schutz des Waldes}

Die Entwicklung wird weiter gehen. Die Bevölkerung wird weiterhin zunehmen; die Industrialisierung dehnt sich aus, der Motorfahrzeugbestand steigt sehr rasch an, der Wasserverbrauch erhöht sich. In Zusammenhang mit dieser Entwicklung können neue Gefahren entstehen oder bestehende Gefährdungen sich verschärfen. Unser Wald ist dazu berufen, die wichtigsten Aufgaben unserer Generation und noch mehr der Zukunft zu erleichtern; er hat lebensentscheidende Schutzfunktionen im weitesten Sinne des Wortes zu erfüllen. Es geht heute nicht in erster Linie darum, Autostraßen zu bauen, neue Flugplätze zu erstellen oder internationale Schiffahrtskanäle anzulegen. Wohl ist die Lösung der Verkehrsfragen von großer Bedeutung; noch wichtiger aber, noch brennender ist es, der Zukunft das saubere Wasser, die reine Luft, die Schönheit der Heimat, Ruhe, Gesundheit und Wohlergehen zu sichern. Wir möchten daher die ganze Bevölkerung 
leidenschaftlich und mit aller Eindringlichkeit $\mathrm{zu}$ einer harten und kompromißlosen Verteidigung unseres Waldes gegen die immer zahlreicher werdenden Rodungsbegehren aufrufen. Tun wir es heute nicht, morgen ist es vielleicht zu spät.

Bester Waldboden besitzt unter den heutigen Verhältnissen und unter der Voraussetzung, daß er weiterhin als Wald bewirtschaftet wird, einen Ertragswert von höchstens 4000 Fr. je Hektar, schlechter Waldboden nur die Hälfte oder ein Viertel davon. Der gleiche Boden in der Nähe der Stadt könnte als Bauland zu 50 Fr. oder 100 Fr. je Quadratmeter, d. h. für 0,5 bis 1 Mio Fr. je ha verkauft werden. In dieser ungeheuren Diskrepanz des Bodenwertes liegt das ganze Problem der Waldrodungen. Würde einem einzelnen Waldeigentümer durch Erteilung einer Rodungsbewilligung ermöglicht, den hundertfachen, ja mehrhundertfachen Wert einer Waldparzelle zu realisieren, dann müßte das bei rechtsgleicher Behandlung auch andern Waldbesitzern gestattet werden. Der Wald wäre dann aber in der Nähe der Siedlungs- und Industriegebiete verloren, gerade da, wo er im allgemeinen Interesse besonders wichtig ist. Daher ist an einer strengen Rodungspraxis festzuhalten. Wohl gehört der Wald seinem Eigentümer, einem Privaten, vielleicht einer Korporation oder einer Gemeinde. Die Landschaft aber, die Natur, Luft und Wasser, unsere Heimat, all das, was mit diesem Wald zusammenhängt, gehört dem ganzen Volk, und dieses ist so sehr auf die Erhaltung der natürlichen Lebensgrundlagen angewiesen, da $\beta$ der Waldeigentümer im öffentlichen Interesse schwerwiegende Eigentumsbeschränkungen, wie es das Rodungsverbot darstellt, in Kauf nehmen muß. Sehr häufig wird die Ansicht vertreten, daß Rodungen dann zulässig seien, wenn für die abgehende Waldfläche durch Aufforstung an einem andern Ort Ersatz geboten werde. Der Wald ist aber nicht eine Holzfabrik, die einfach abgebrochen und an einem andern Ort wieder aufgestellt werden könnte. Durch die Rodung wird Waldbestand, dessen Aufbau Jahrzehnte, ein Jahrhundert oder noch länger dauerte und die ganze vielfältige Lebensgemeinschaft zerstört. Bis anderseits die Neuaufforstung Wald im ökologisch-biologischen Sinn wird und bis die großartige Architektur von Waldboden am neuen Standort geschaffen ist, wie sie gerade für den Wasserhaushalt besonders wirksam ist, vergeht eine, vergehen zwei Baumgenerationen. Außerdem ergäbe sich eine unerwünschte Verlagerung des Waldes, denn die Rodungsbegehren werden vorwiegend in Gebieten mit starker Bautätigkeit gestellt, während die Ersatzaufforstungen in der Regel in abgelegenen, meist ohnehin schon stark bewaldeten Gegenden angeboten werden. Der Bundesrat hat daher in einem Rekursentscheid betreffend Ablehnung eines Rodungsgesuches durch den zürcherischen Regierungsrat mit Recht festgehalten, daß mit jeder Waldrodung der Bevölkerung Wohlfahrtswirkungen verlorengehen, die durch Aufforstungen in abgelegenen Gegenden nicht ersetzt werden können.

In stark entwaldeten ausländischen Staaten und in überseeischen Gebieten investiert man heute riesige Mittel, um den Wald wieder zu schaffen. Jugoslawien forstet unter unsäglichen Schwierigkeiten im Karst kahle, ausgewaschene Hänge auf. In Israel wird mit ungeheurem Aufwand versucht, in öden, steinigen und sandigen Gebieten wieder Wälder aufzubringen und den Wasserhaushalt zu verbessern. Frankreich, Spanien, Portugal haben in den letzten Jahren ausgedehnte, neue Wälder angepflanzt, und die Vereinigten Staaten von Nordamerika bekämpfen mit einem Budget von jährlich über 2 Milliarden Franken die wegen der starken Entwaldung vor sich gehende Erosion des Bodens durch Wasser und Wind. Es ist vorauszusehen, daß unter dem Einfluß der Überschwemmungskatastrophen vom November 1966 in Italien ein umfangreiches Aufforstungsprogramm aufgestellt wird, um weitere derartige Hochwasser durch bessere Bewaldung der kahlen Einzugsgebiete zu vermeiden oder doch zu mildern. Wie viel einfacher ist es bei uns, wo der Wald noch da ist! Wir brauchen ihn nur zu erhalten und zu pflegen. Leider ist heute die sorgfältige Bewirtschaftung des Waldes wegen der sich ständig verschlechternden Ertragslage unserer Waldwirtschaft gefährdet; damit ist die Erhaltung und Förderung seiner Schutz- und Wohlfahrtswirkungen bedroht.

Es ist eine schöne, wenn auch schwere Aufgabe, den Wald gegen die zahlreichen Angriffe, denen er ausgesetzt ist, zu verteidigen. Dankbar erkennen wir, $\mathrm{da} ß$ uns immer weitere Kreise der Bevölkerung, viele Behörden und maßgebende Leute in diesem Kampf vorbehaltlos unterstützen. Seien wir uns unserer Verantwortung der Zukunft gegenüber bewußt. Schützen wir den Wald, denn er schützt uns! "Wir sollten endlich begreifen, daß es kein besseres Kleid der Erde gibt als den Wald" (Barbe-Baker in «Grüne Herrlichkeit»).

Hirschgeweihfarn als Epiphyt an Baumstamm. Photo René Gardi.

Originalfarbbild aus «Der Wald», Kümmerly \& Frey, Geographischer Verlag, Bern. 


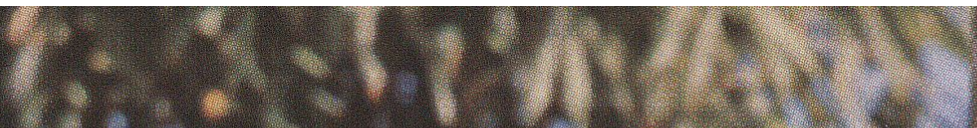

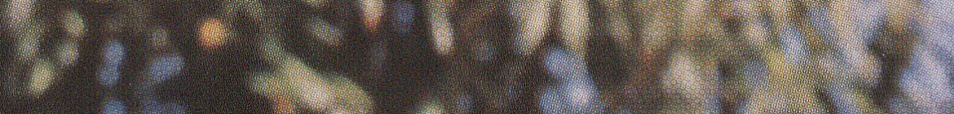
25.0

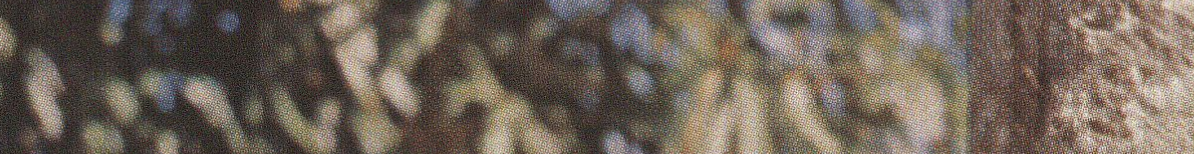
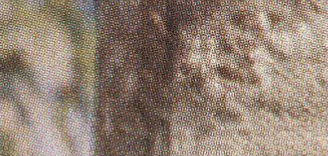

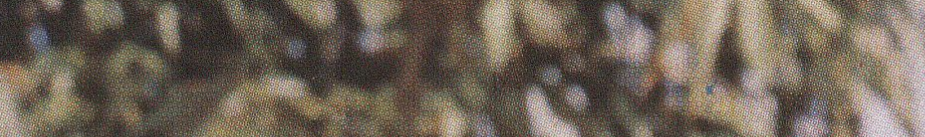

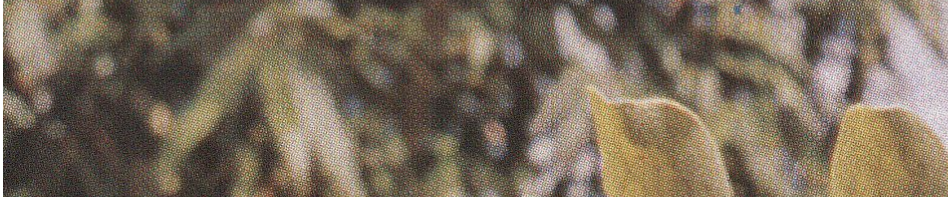

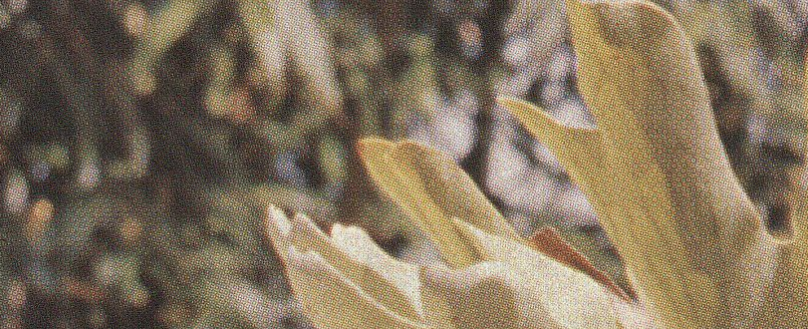

isciant

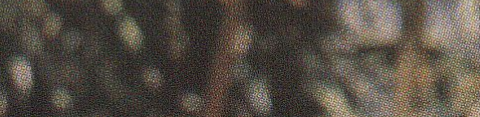

"xis

1)

Avations

xist

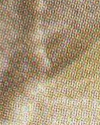

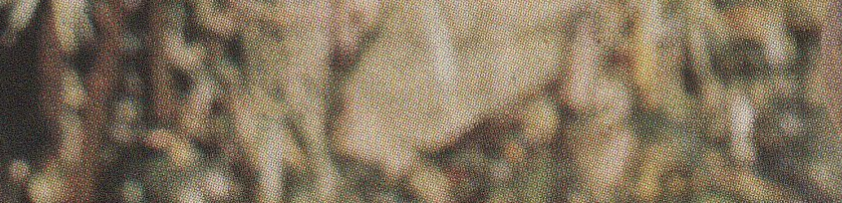
at onds

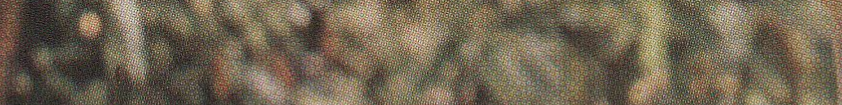

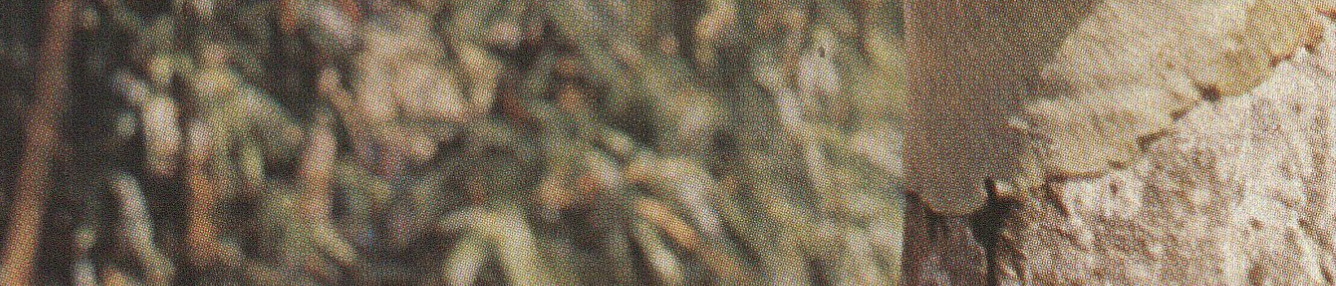
3hy 